\title{
Healthcare worker knowledge and skills following coaching in WHO early essential newborn care program in the Solomon Islands: a prospective multi-site cohort study
}

Shidan Tosif ${ }^{*}$ (D), Anna Jatobatu ${ }^{2}$, Anita Maepioh ${ }^{3}$, Amy Gray ${ }^{1}$, Howard Sobel $^{4}$, Priya Mannava ${ }^{5}$ and Trevor Duke $^{1}$

\begin{abstract}
Background: Newborn mortality in Oceania declined slower than other regions in the past 25 years. The World Health Organization (WHO) introduced the Early Essential Newborn Care program (EENC) in 2015 in Solomon Islands, a Small Island Developing State, to address high newborn mortality. We explored knowledge and skills retention among healthcare workers following EENC coaching.
\end{abstract}

Methods: Between March 2015 and December 2017, healthcare workers in five hospitals were assessed: pre- and post-clinical coaching and at a later evaluation. Standardised written and clinical skills assessments for breathing and non-breathing baby scenarios were used. Additionally, written surveys were completed during evaluation for feedback on the EENC experience.

Results: Fifty-three healthcare workers were included in the evaluation. Median time between initial coaching and evaluation was 21 months (IQR 18-26). Median written score increased from 44\% at baseline to 89\% post-coaching $(p<0.001)$, and was $61 \%$ at evaluation $(p<0.001)$. Skills assessment score was $20 \%$ at baseline and $95 \%$ postcoaching in the Breathing Baby scenario $(p<0.001)$. In the Non-Breathing Baby scenario, score was $63 \%$ at baseline and $86 \%$ post-coaching $(p<0.001)$. At evaluation, median score in the Breathing Baby scenario was $82 \%$ a reduction of $13 \%$ from post-coaching $(p<0.001)$ and $72 \%$ for the Non-Breathing Baby, a reduction of $14 \%$ postcoaching $(p<0.001)$. Nurse aides had least reduction in evaluation scores of $-2 \%$ for the Breathing Baby and midwives $-10 \%$ for the Non-Breathing Baby respectively from post-coaching to evaluation.

Conclusions: EENC coaching resulted in immediate improvements in knowledge and skills but declined over time. Healthcare workers who used the skills in regular practice had higher scores. Complementary quality improvement strategies are needed to sustain resuscitation skills following training over time.

Trial registration: Australia New Zealand Trial Registry, Retrospective Registration (12/2/2019), registration number ACTRN12619000201178.

Keywords: Neonatal resuscitation, Neonatal training programs, Low- and middle-income countries, Small Island developing states

\footnotetext{
* Correspondence: shidan.tosif@gmail.com

${ }^{1}$ Centre for International Child Health, University of Melbourne, Murdoch

Children's Research Institute, Royal Children's Hospital Melbourne, 50

Flemington Rd, Parkville, VIC 3052, Australia

Full list of author information is available at the end of the article
}

(c) The Author(s). 2020 Open Access This article is distributed under the terms of the Creative Commons Attribution 4.0 International License (http://creativecommons.org/licenses/by/4.0/), which permits unrestricted use, distribution, and reproduction in any medium, provided you give appropriate credit to the original author(s) and the source, provide a link to the Creative Commons license, and indicate if changes were made. The Creative Commons Public Domain Dedication waiver (http://creativecommons.org/publicdomain/zero/1.0/) applies to the data made available in this article, unless otherwise stated. 


\section{Background}

The Oceania region (excluding Australia and New Zealand) experienced the slowest rate of reduction in neonatal mortality compared to other developing regions during the period of the Millennium Development Goals [1]. Like other lower-middle income Small Island Developing States (SIDS) [2], the small health workforce in the Solomon Islands faces enormous accessibility issues including dispersed populations, challenging geographies and environmental vulnerability to natural disasters.

Many gaps exist in the health facility care of common causes of neonatal mortality: intrapartum-related complications, complications of prematurity and severe infection $[3,4]$. Neonatal resuscitation training in facilities can reduce early deaths $[3,5,6]$. Supportive interventions in the immediate newborn period such as skin-to-skin contact, early and exclusive breastfeeding and temperature control have been shown to improve outcomes [7]. A combined approach including early essential newborn care, resuscitation, and basic care of common neonatal problems could address the most common causes of perinatal mortality [8].

Continuing professional development for health care workers in Solomon Islands presents challenges due to few opportunities to learn or refresh knowledge and skills, and this is a barrier to improving newborn quality of care. The World Health Organisation (WHO) Early Essential Newborn Care (EENC) program focuses on developing skills and knowledge for the management of "Breathing" and "Non-breathing" babies. It addresses the critical first moments and days of newborn care for healthcare workers in health facilities, where over 95\% of births take place in the Western Pacific [9-11]. Key content areas are neonatal resuscitation and basic newborn care, with emphasis on thorough drying, delayed cord clamping, skin-to-skin contact, reduction of harmful practices and support for exclusive breastfeeding. Whilst these measures do not require sophisticated technology, they rely on healthcare worker skills and knowledge to reduce risk of infection or death around the time of birth [4].

The aim of this study was to determine the impact on skills and knowledge amongst healthcare workers following EENC training, and characterise the healthcare provider attributes that effect retention and practice in the unique healthcare context of Solomon Islands.

\section{Methods}

\section{Description of intervention}

EENC was implemented as the country-level program for newborn care in Solomon Islands. The Ministry of Health and Medical Services (MHMS) rolled out the program across the nine provinces in the country from 2015 onwards. Following a national training, a newborn nurse coordinator (AJ) delivered subsequent trainings at the National Referral Hospital (NRH) and provincial hospitals with 1-2 nurse or midwife co-facilitators. The 2-day EENC program consisted of on-site coaching, with a low participant to facilitator ratio (6:1) [11]. The venue for training was the delivery room, or, where not possible, a room setup with a similar arrangement. The coaching approach was participatory in style without didactic teaching. In small groups, a participant would first demonstrate normal practice. The facilitators then explored why certain actions were taken, with discussion of the evidence for correct practice, as well as the evidence that some practices are unnecessary or potentially harmful (e.g. unnecessary routine suctioning, early washing the baby, separation of the baby and the mother). Participants were invited to share feedback in a supportive way, pointing out correct actions or if improvement was required. Each participant then took part in repeated practice whilst the facilitator used the EENC skills checklist as a reference. At the end of the coaching, the establishment of a quality improvement team within 3 months was planned at each site, with relevant guidance provided from EENC modules.

\section{Setting}

Solomon Islands shares many geographical and demographic characteristics with other low- and middle-income countries in Oceania and SIDS globally. A population of almost 600,000 is dispersed amongst nine provinces and more than 900 islands. For this study, the Solomon Islands MHMS purposively selected five hospitals which together serve $80 \%$ of the national population: the NRH, Gizo Hospital, Kil'ufi Hospital, Makira Hospital, and Good Samaritan hospitals.

\section{Study design and participants}

We conducted a pre and post, multiple-site, facilitybased study between March 2015 and November 2017 . Eligible participants were health care workers who attended EENC coaching and were evaluated prior to refresher training.

The primary outcome measures were knowledge and simulated skill scores according to standardised assessments contained in EENC, as have been used in the implementation of EENC throughout the Western Pacific region [12] and are available online [11]. Baseline scores for knowledge and skills were established from precoaching assessment of one random participant in each group. Assessment of all participants occurred immediately following EENC coaching, and prior to a refresher (see Fig. 1 for timeline). Timing of refresher was aimed for 12-months post-coaching.

Knowledge was assessed with a written test, with questions on the themes of routine newborn care, breast- 


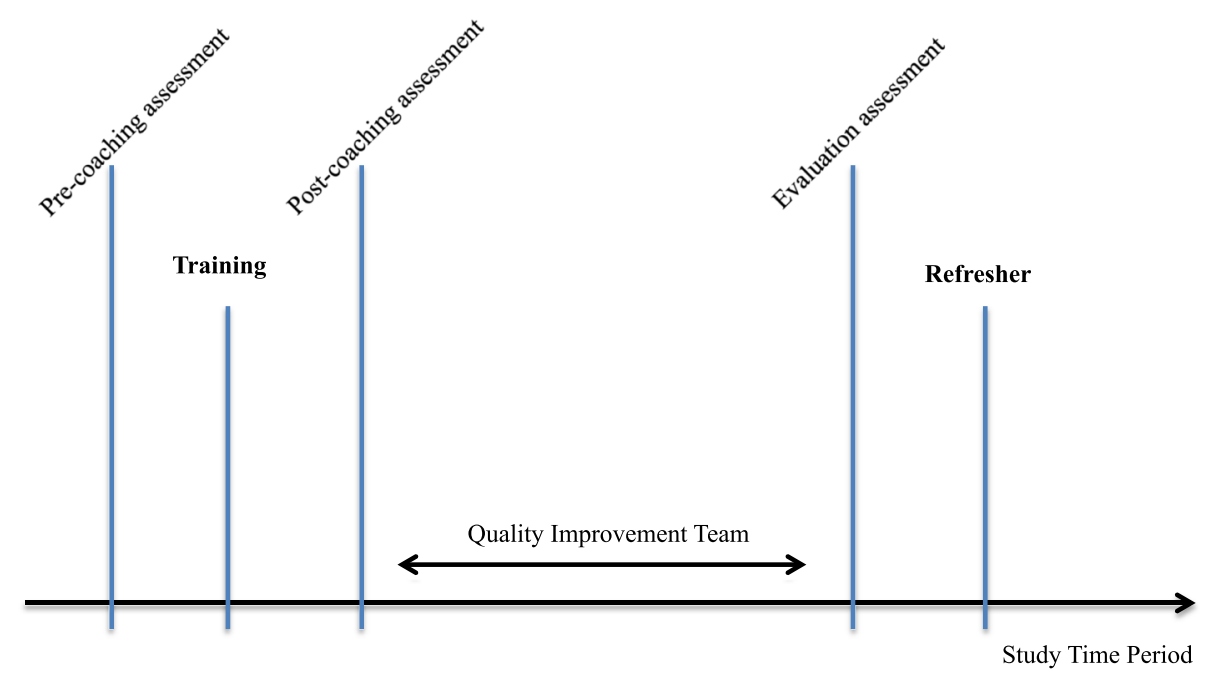

Fig. 1 Sequence of study period activities

feeding and resuscitation. The skills assessment tested skills in managing a mother and newborn in two scenarios, the delivery of a "Breathing" and "Non-breathing" baby. The written test and scenarios were carried out in a room set-up for the purpose under examination conditions.

Demographic data was collected using a confidential written questionnaire including experience, qualifications, work location, and attendance at other neonatal training (see Additional file 1). We asked participants about their satisfaction of the content of EENC using a 5-point Likert scale $(1=$ Too complicated, $2=$ A little complicated, $3=$ Just right, $4=$ A little simple, $5=$ Too simple). Opportunity was given to clarify questions using Solomon Islands Pigin during the written component and skills assessment scenarios were carried out in Solomon Islands Pigin or English according to participant preferences. The assessment team consisted of midwife (AM), newborn nurse coordinator $(\mathrm{AJ})$ and visiting paediatrician $(\mathrm{ST})$.

\section{Analysis}

Data were summarised with frequencies or percentages for categorical values, or means and standard deviations (SD) or medians and interquartile ranges (IQR) for continuous variables. Difference testing between precoaching and post-coaching groups was performed using Mann-Whitney U test. Comparison between pairs of scores from health care workers between post-coaching to refresher was performed using the Paired Sign Test. Testing for differences of continuous variables between groups was performed using the Kruskal-Wallis test.

Written and skill assessment scores were manually entered from data collection forms into Excel, before cleaning and analysis in STATA (Version 15.0). This study was approved by the Solomon Islands Health
Research and Ethics Review Board (project number HRE033/16) and the University of Melbourne Human Research Ethics Committee (HREC number 1646267.1). This study adheres to STROBE guidelines for reporting observational studies [13].

\section{Results}

A total of 53 participants were included. Pre-coaching baseline scores were established from 25 participants in the Breathing Baby scenario, and 15 participants in the Non-Breathing Baby scenario. Median time for participants between coaching and evaluation was 21 months (IQR 18-26). There were 23 nurses, 15 doctors, 8 midwives and 7 nurse aides. The average healthcare worker was 36 years of age ( \pm 12 years), and had 9 years experience in neonatal care ( \pm 7 years). Six-per cent (3/53) of healthcare workers participated in a quality improvement activity following training. Thirty-one per cent $(16 / 53)$ of healthcare workers had worked in two or more health facilities in the last 5 years. Forty-seven per cent $(25 / 53)$ of healthcare workers had past training related to newborn care, none had received a refresher or follow up training previously. Past trainings was in Integrated Management of Childhood Illnesses, World Health Organisation Hospital Care for Children, Mother Baby Friendly Hospital Initiative, University based or other visiting programmatic trainings in newborn care.

\section{Evaluation of written scores}

All healthcare workers participated in the written test $(n=53)$ (Fig. 2). There was a significant increase in written scores immediately following coaching. Median written scores increased $45 \%$ from $44 \%$ (IQR 33-56) to $89 \%$ (IQR 78-94) $(p<0.001)$. At the time of the evaluation, median scores were $61 \%$, (IQR 50-72) a reduction 


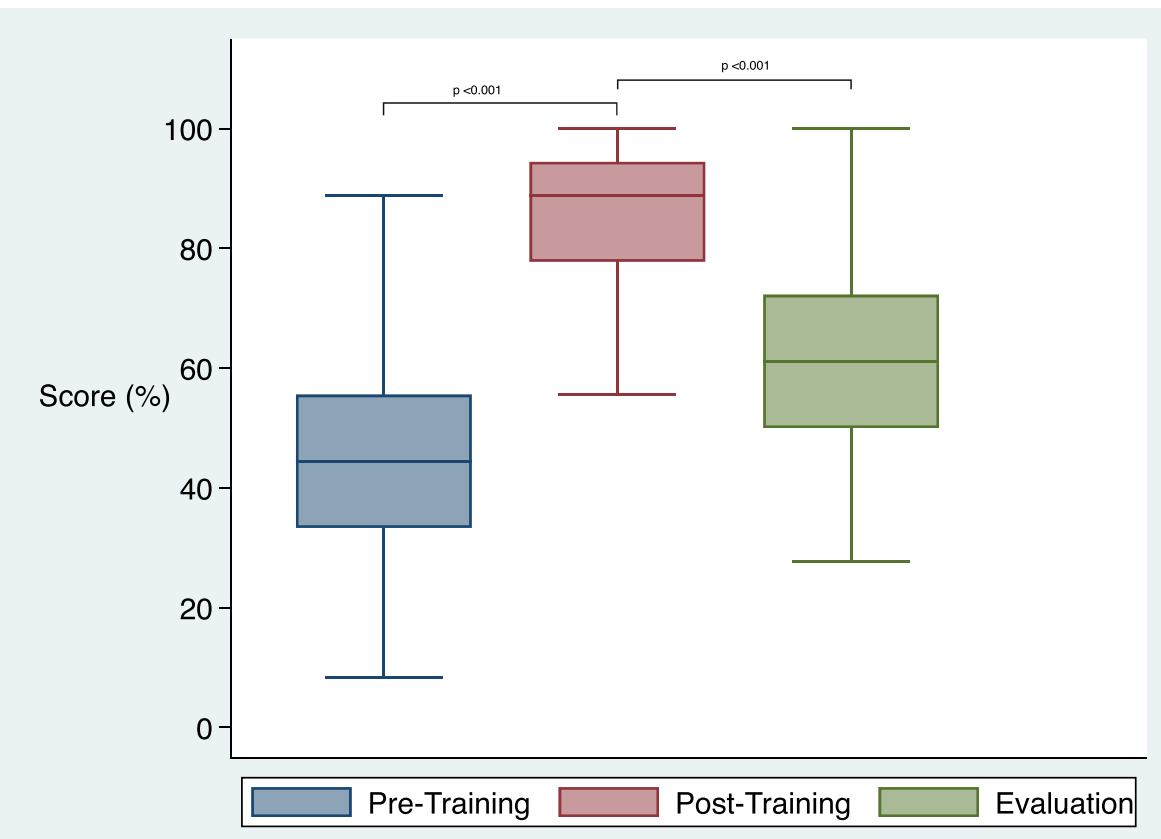

Fig. 2 Written score assessment results by time period

of $28 \%(p<0.001)$ from post-coaching levels but still $17 \%$ higher than at baseline.

\section{Evaluation of skills}

Median scores in the Breathing Baby scenario went from 20\% (IQR 11-32) pre-coaching, to 95\% (IQR 91-95) post-coaching, and $82 \%$ (IQR 66-91) at time of evaluation $(p<0.001)$ (Fig. 3). Median scores in the Non-Breathing Baby scenario were 63\% (IQR 45-73) pre-coaching, $86 \%$ (IQR $86-88$ ) post-coaching and $72 \%$ (IQR 63-81) at evaluation $(p<0.001)$ (Fig. 4). In the Breathing Baby scenario, health care workers who

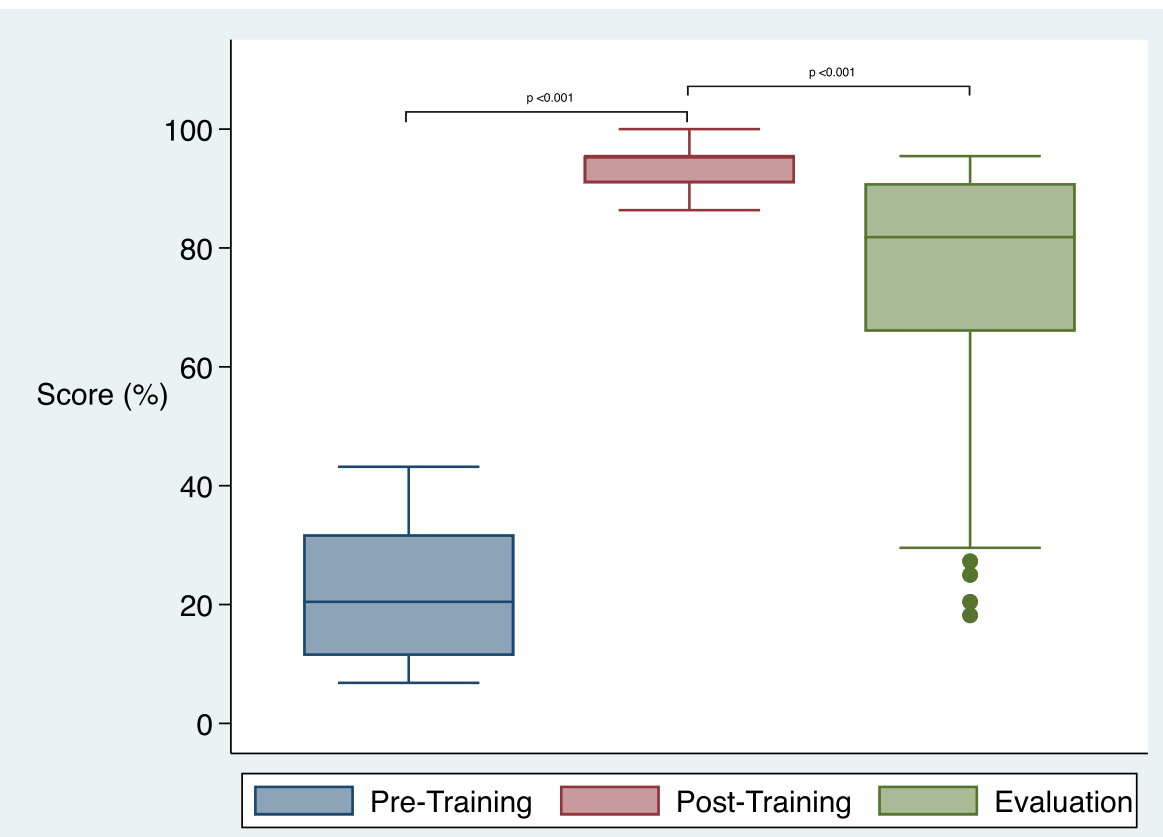

Fig. 3 Skill scores in breathing baby scenario by time period 


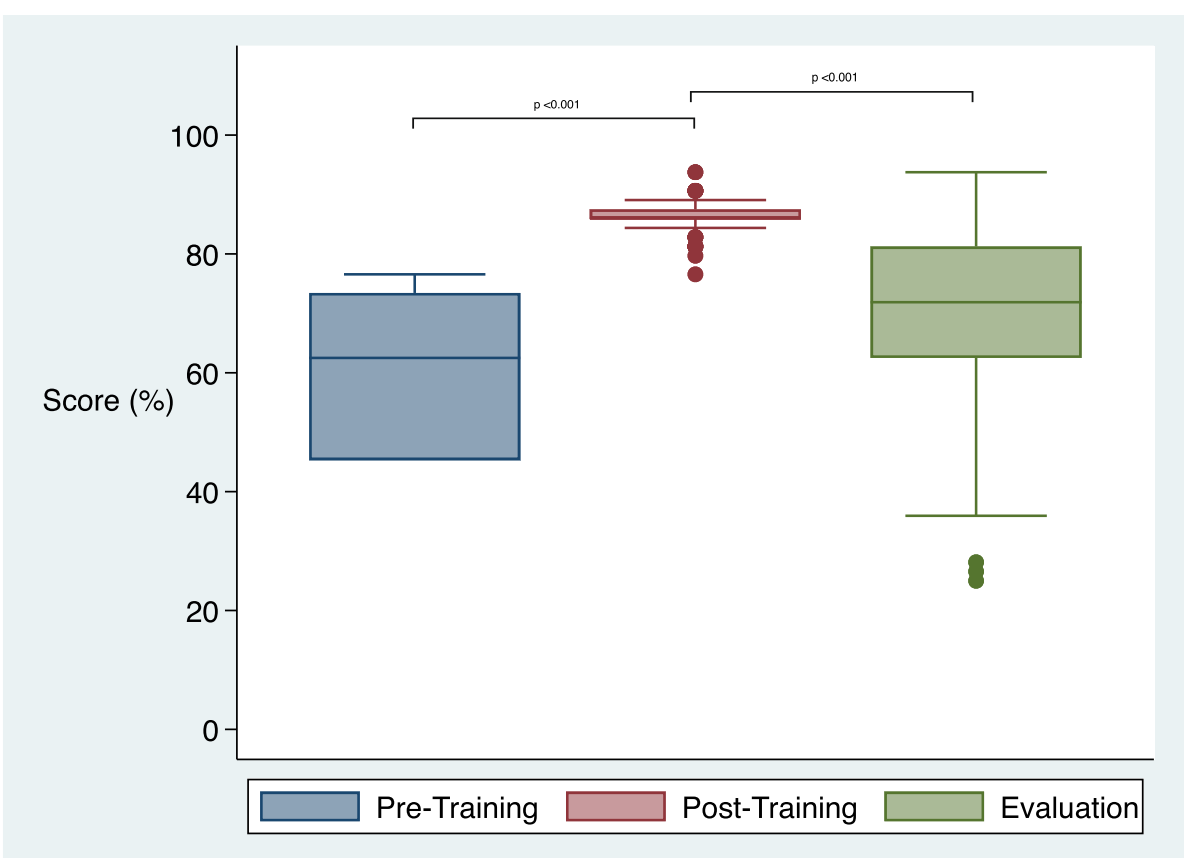

Fig. 4 Skill scores in non-breathing baby by time period

received past training did not have better retention than those who had not received training $(-20 \%$ and $-14 \%$ respectively, $p=0.291$ ), and had poorer performance in the Non-Breathing Baby scenario $(-13 \%$ vs $-22 \%, p=$ 0.040 ).

In the Non-Breathing Baby scenario, mean scores for neonatal resuscitation skills were lower than routine pre-birth preparations, immediate newborn care, and post-partum care: $66 \%$ vs 80,72 and $74 \%$ respectively (see Table 1).

\section{Skill retention by cadre}

When separated by cadre of healthcare worker, nurse aides showed the best skill retention in the breathing baby scenario, and midwives in the non-breathing baby scenario when post-coaching and evaluation scores were compared (Figs. 5 and 6). In the breathing baby scenario, nurse aides had a median score reduction of $-2 \%$ (IQR -18 to 2 ), midwives $-10 \%$ (IQR -16 to -1 ), nurses $-11 \%$ (IQR -43 to 0 ) doctors $-18 \%$ (IQR 31 to -4$)(p=0.237)$. In the non-breathing baby scenario, midwives had a median score difference of $-8 \%(-13$ to 0$)$, nurses $-13 \%(-30$ to -5$)$, doctors $-19 \%$ (IQR -27 to -6 ) and nurse aides $-22 \%$ (IQR -27 to 0$)(p=0.440)$.

\section{EENC feedback}

Most participants found the content in the EENC program "just right" (83\%). 7\% found the course "a little too complicated", 5\% "a little simple", and 5\% "too simple".
The mean Likert Scale score response was 3 (SD 0.5) equating to "just right".

\section{Discussion}

The 2-day EENC coaching intervention delivered by a small team of Solomon Islands Ministry of Health staff in a low-resource Pacific Island setting, increased knowledge by $45 \%$, and skills by $75 \%$ (Breathing Baby) and $23 \%$ (Non-Breathing baby) respectively. At evaluation after a median of 21 months post-training, skills experienced a modest reduction to $82 \%$ (Breathing Baby) and 72\% (NonBreathing Baby). Skills related to pre-birth preparation and immediate post-partum care were better retained than for neonatal resuscitation. Nurse aids and midwives who were engaged in providing routine neonatal care had least reductions in scores at evaluation. Health professionals who did not use the skills routinely had greater reductions in scores at the time of evaluation, but still remained significantly above baseline. The EENC program content was well received by participants.

These findings may have implications for the design and implementation of newborn training in other similar settings. The EENC program uses a practical, coaching methodology with a focus on two practical scenarios using minimal resources, without didactic or excess content over a 2-day training period. Case based learning and clinical simulations with frequent repetition have been shown to be effective educational methods for health care worker training [14]. Short training periods limit time away from clinical posts and are less 
Table 1 Mean participant scores for each skill checklist item for the Non-Breathing Baby scenario

\begin{tabular}{|c|c|}
\hline & $\begin{array}{l}\% \text { Correct } \\
\text { Demonstration } \\
(n=53)\end{array}$ \\
\hline \multicolumn{2}{|l|}{ Pre-birth preparations } \\
\hline Checked room temperature; turned off fans & 80 \\
\hline Washed hands (first of two) & 84 \\
\hline Dry cloth placed on mother's abdomen & 78 \\
\hline Prepared the newborn resuscitation area & 82 \\
\hline Checked if bag and mask are functional & 78 \\
\hline Washed hands (second of two) & 78 \\
\hline Wore two pairs of clean gloves & 89 \\
\hline Put forceps, cord clamp/ties in easy-to-use order & 71 \\
\hline Mean & 80 \\
\hline \multicolumn{2}{|l|}{ Immediate newborn care } \\
\hline Call out time of birth (hours, minutes, seconds) & 73 \\
\hline Drying was started within $5 \mathrm{~s}$ after birth & 80 \\
\hline $\begin{array}{l}\text { Dried the baby thoroughly (wiped the eyes, } \\
\text { face, head, front, back, arms and legs) }\end{array}$ & 82 \\
\hline Removed the wet cloth & 66 \\
\hline Baby was in direct skin-to-skin contact & 68 \\
\hline Covered baby's body and head with dry cloth & 64 \\
\hline Mean & 72 \\
\hline \multicolumn{2}{|l|}{ Neonatal resuscitation } \\
\hline Called for help & 51 \\
\hline Remove first pair of gloves & 47 \\
\hline Quickly clamped and cut cord & 85 \\
\hline Moved baby to resuscitation area & 69 \\
\hline Cover baby quickly during and after transfer & 67 \\
\hline Positioned head correctly to open airways & 72 \\
\hline $\begin{array}{l}\text { Applied face mask firmly over chin, mouth } \\
\& \text { nose }\end{array}$ & 67 \\
\hline Chest rise within 1 min of birth & 60 \\
\hline Squeezed bag to give $30-50$ breaths per minute & 57 \\
\hline $\begin{array}{l}\text { Maintained good chest rise throughout or } \\
\text { took steps to improve ventilation }\end{array}$ & 64 \\
\hline $\begin{array}{l}\text { Once baby's breathing well, stopped mechanical } \\
\text { ventilation }\end{array}$ & 89 \\
\hline Mean & 66 \\
\hline \multicolumn{2}{|l|}{ Immediate postpartum care } \\
\hline Returned to skin to skin contact, covered baby & 90 \\
\hline Checked for another baby & 69 \\
\hline Gave oxytocin to the mother & 77 \\
\hline Delivered placenta & 53 \\
\hline $\begin{array}{l}\text { Counselled mother that baby is ok and on } \\
\text { feeding cues }\end{array}$ & 83 \\
\hline Mean & 74 \\
\hline
\end{tabular}

expensive. This program was implemented through the actions of a small team dedicated to a large geographical area with little opportunities to follow up. More strategies for implementing programs in such settings with limited resources are needed.

Our study found better retention of skills and knowledge in midwives and nurse aides compared to other cadres, reflective of other studies where birth volume and associated frequent clinical practice was associated with better retention [15]. Of concern was the relatively higher drop-off of scores for doctors in both scenarios. Doctor's skills are often deferred to in resuscitation or for the care of critically ill neonates. For health care workers who infrequently attend deliveries, periodic refreshers and regular practice with portable simulators can maintain skills $[16,17]$. Consideration needs to be given to strategies that fit the local context, particularly those that have feasibility in the confines of geography and financial limitations. These could include requirements for continuing education, strengthening local rather than national coordinator roles or providing incentives.

High post-coaching scores in this study demonstrate effectiveness of the training approach in improving knowledge and skills, a result similar to other studies, which range from improvements of between 7 to $89 \%$ from baseline [18]. Knowledge and skill improvements are also reflected in national surveys identifying higher rates of adherence to skin-to-skin initiation and duration [19]. The higher Non-Breathing Baby pre-coaching scores compared with Breathing Baby scores are likely due to a boosted score, from this assessment taking place second, with participants incorporating newly learnt skills from the first scenario. Knowledge and skills fall-off occurred in half of 10 studies identified in a systematic review on this theme, with three showing no fall-off and two with a range of retention according to cadres of birth attendants and prior training exposure [18]. Knowledge and skills fall off in the range described in our study has been reported following neonatal training programs [18, 20, 21] and has led to the addition of quality improvement approaches [22] incorporating various strategies such as daily bag and mask skills practice, peer review and weekly skills checklist to improve retention [23]. Whilst establishment of a quality improvement team within 3 months of coaching was recommended with relevant guidance in the EENC modules, they were not utilised at the hospital level in contrast to other countries in the region using EENC where quality improvement implementation occurred up to half the facilities $[19,24]$. This was likely due to the small training team in Solomon Islands not being available to facilitate, mentor and support participants from remote geographical locations, with visits occurring not more than once per year due to financial and logistical challenges. 


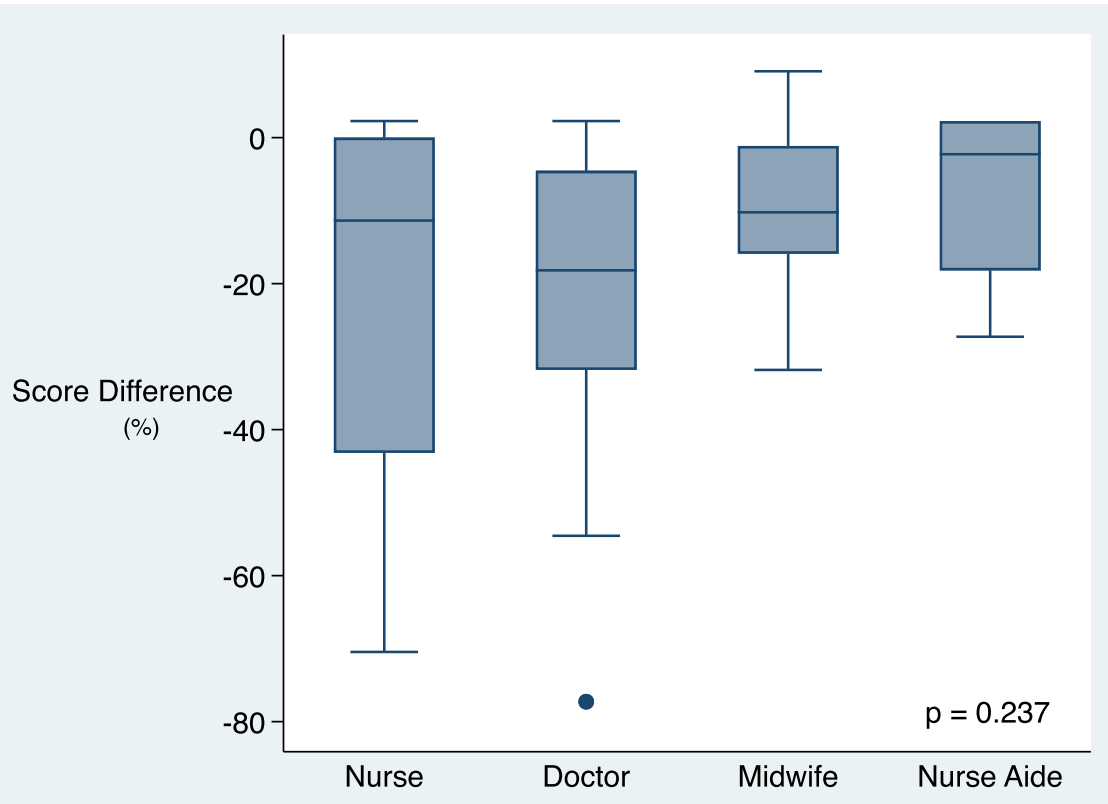

Fig. 5 Score difference (post-coaching vs evaluation) in breathing baby scenario by cadre

Further analysis of the skills demonstrated showed that resuscitation skills, specifically those related to bag and mask ventilation, were retained less than skills for routine care. This may be due to the lower frequency of resuscitation events in practice, and the greater degree of technical expertise required. An analysis of facilitator and learner perceptions from a neonatal resuscitation program across two low- and middle-income countries found additional training was required to establish resuscitation skills and recommended continued learning and active mentoring to establish this practice [25]. An intervention for Helping Babies Breathe in Nepal, used a

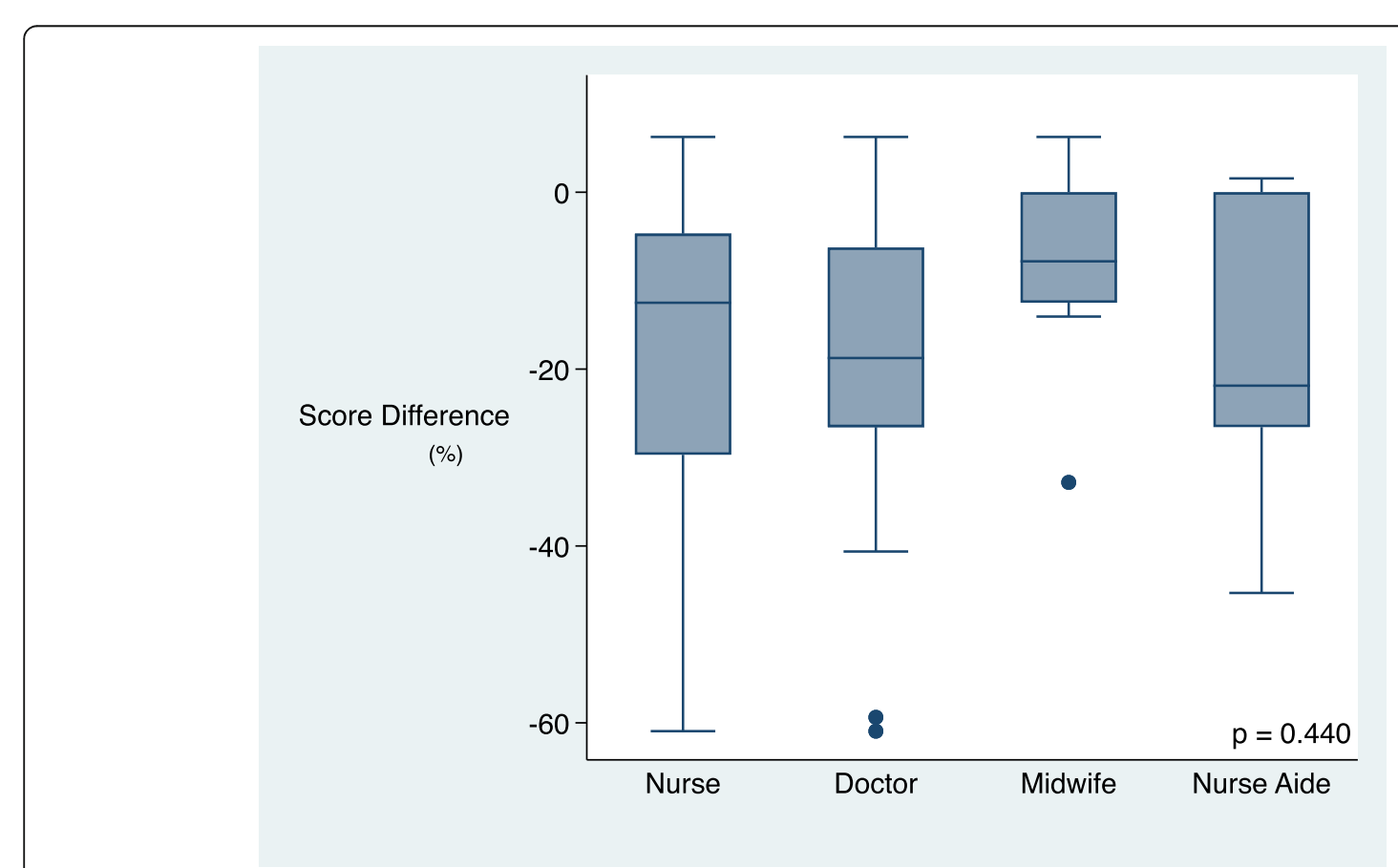

Fig. 6 Non-breathing baby scenario score difference (post training vs evaluation) by cadre 
neonatal resuscitation protocol which focused on bag and mask ventilation included training, daily skill checks, preparation for resuscitation at every birth, self-evaluation and peer review [23]. Skills and competencies in care of high-risk babies improved following supportive supervision and monitoring in Uganda [26]. Through additional exposure, these additional measures may assist in resuscitation through familiarisation with a typically stressful, low frequency event, of higher complexity.

This study has some limitations. There was no comparison group, and time between coaching and evaluation varied due to logistics and accessibility. Only one participant from each coaching group was assessed at baseline. This reflected a pragmatic approach, where limited resources and time for the coaching were balanced with research needs. Some of the participants in this study had prior neonatal training but none had additional newborn training other than EENC during the study period. Our study used knowledge and simulation to assess healthcare worker skill performance. Ideally, evaluation of training programs would be through measuring impact on neonatal morbidity and mortality, and neurodevelopmental outcomes [10]. However, in practice, these outcomes are difficult to measure due to the sample sizes required, and a research infrastructure that is out of reach of small teams on low budgets. Assessments of knowledge and skills provide indirect information on effectiveness of neonatal resuscitation programs [10] and a systematic review identified four out of five studies with a positive correlation between simulated test scores and clinical behaviour [18]. Other training programs have used similar assessment time points in assessing knowledge and skills [27].

\section{Conclusion}

The EENC coaching program was implemented by a small team and resulted in improved knowledge and skills, especially among those who performed immediate newborn care routinely; however there was a fall-off within 18 months especially among those who did not use the skills routinely. Routine newborn skills were sustained more than resuscitation skills. Complementary strategies are needed to sustain resuscitation skills following coaching over time with novel methods required to reach remote health workers who have infrequent opportunities for resuscitation practice.

\section{Supplementary information}

Supplementary information accompanies this paper at https://doi.org/10. 1186/s12884-020-2739-z.

Additional file 1. Written Survey. Written Questionnaire for participant background, experience and training satisfaction scores.

\section{Abbreviations}

EENC: Early Essential Newborn Care; IQR: Inter Quartile Range: MHMS: Ministry of Health and Medical Services; SIDS: Small Island Developing State; WHO: World Health Organization

\section{Acknowledgements}

The authors would like to thank all the health care workers, the staff at the Department of Paediatrics at the National Referral Hospital Honiara and Ministry of Health and Medical Services.

\section{Authors' contributions}

Conception of the study: ST, TD; data collection: ST, AJ, AM; data analysis and interpretation: ST, AJ, AM, TD; drafting the article: ST; critical revision of the article: $A J, A M, A G, H S, P M, T D$; final approval of the version to the published: ST, AJ, AM, AG, HS, PM, TD.

\section{Funding}

The authors received no funding for the design, interpretation or composition of the manuscript.

\section{Availability of data and materials}

According to the regulations of the University of Melbourne, the dataset generated and analysed during the study, cannot be made public. The data are however available from the corresponding author (ST) for inspection upon reasonable request.

\section{Ethics approval and consent to participate}

This study was approved by the Solomon Islands Health Research and Ethics Review Board (project number HRE033/16) and the University of Melbourne Human Research Ethics Committee (HREC number 1646267.1). Written consent was obtained from all participants to participate in this study.

\section{Consent for publication}

Not applicable.

\section{Competing interests}

Dr. Howard Sobel and Ms. Priya Mannava are employed by the World Health Organisation.

\section{Author details}

${ }^{1}$ Centre for International Child Health, University of Melbourne, Murdoch Children's Research Institute, Royal Children's Hospital Melbourne, 50 Flemington Rd, Parkville, VIC 3052, Australia. ${ }^{2}$ National Newborn Health Coordinator, Reproductive and Child Health Department, Ministry of Health and Medical Services, Honiara, Solomon Islands. ${ }^{3}$ Department of Obstetrics and Gynaecology, National Referral Hospital, Honiara, Solomon Islands. ${ }^{4}$ Coordinator Maternal, Child Health and Quality and Safety, World Health Organization Regional Office for the Western Pacific, Manila, Philippines.

${ }^{5}$ Technical Officer, Surveillance, Information and Accountability, Reproductive, Maternal, Newborn, Child and Adolescent Health, World Health Organisation Regional Office for the Western Pacific, Manila, Philippines,

Received: 18 February 2019 Accepted: 14 January 2020

Published online: 07 February 2020

\section{References}

1. United Nations Children's Fund. Level and Trends in Child Mortality Report 2017. 3 UN Plaza, New York, New York, 10017 USA 2017.

2. The World Bank. Solomon Islands Country Information 2016 [updated 2017 : cited 2018 April 11th]. Available from: https://data-worldbank-org.ezp.lib. unimelb.edu.au/country/solomon-islands.

3. Lawn JE, Blencowe H, Oza S, You D, Lee AC, Waiswa P, et al. Every newborn: progress, priorities, and potential beyond survival. Lancet. 2014;384(9938): 189-205.

4. Sobel HL, Silvestre MA, Mantaring JB 3rd, Oliveros YE, Nyunt US. Immediate newborn care practices delay thermoregulation and breastfeeding initiation. Acta Paediatr. 2011;100(8):1127-33.

5. Lee AC, Cousens S, Wall SN, Niermeyer S, Darmstadt GL, Carlo WA, et al. Neonatal resuscitation and immediate newborn assessment and stimulation for the prevention of neonatal deaths: a systematic review, meta-analysis and Delphi estimation of mortality effect. BMC Public Health. 2011;11(3):S12. 
6. Deorari AK, Paul VK, Singh M, Vidyasagar D. Impact of education and training on neonatal resuscitation practices in 14 teaching hospitals in India. Ann Trop Paediatr. 2001;21(1):29-33.

7. Tran HT, Mannava P, Murray JCS, Nguyen PTT, Tuyen LTM, Hoang Anh T, et al. Early essential newborn care is associated with reduced adverse neonatal outcomes in a tertiary Hospital in Da Nang, Viet Nam: a pre- postintervention study. EClinicalMedicine. 2018;6:51-8.

8. Wall SN, Lee AC, Niermeyer S, English M, Keenan WJ, Carlo W, et al. Neonatal resuscitation in low-resource settings: what, who, and how to overcome challenges to scale up? Int J Gynaecol Obstet. 2009;107 Suppl 1: S47-62 S3-4.

9. WHO. Action plan for healthy newborn infants in the Western Pacific region (2014-2020). Geneva: Available online: https://www.unicef.org/eapro/ regional_action_plan_new_born_infants.pdf [last accessed May 2017]; 2014.

10. Narayanan IRM, Cordero D, Faillace S, Sanghvian T. The Components of Essential Newborn Care. Arlington, Virginia: Basics Support for Institutionalizing Child Survival Project (BASICS II) for the United States Agency for International Development; 2004.

11. World Health Organisation Western Pacific Region. Coaching for the first embrace: facilitators guide (early essential newborn care). World Health Organization regional Office for the Western Pacific, editor. Geneva, Switzerland: WHO Press; 2016.

12. Obara $\mathrm{H}$, Sobel H. Quality maternal and newborn care to ensure a healthy start for every newborn in the World Health Organization Western Pacific Region. BJOG. 2014;121 Suppl 4(4):154-9.

13. von Elm E, Altman DG, Egger M, Pocock SJ, Gotzsche PC, Vandenbroucke $J P$. The strengthening the reporting of observational studies in epidemiology (STROBE) statement: guidelines for reporting observational studies. J Clin Epidemiol. 2008;61(4):344-9.

14. Bluestone J, Johnson P, Fullerton J, Carr C, Alderman J, BonTempo J. Effective in-service training design and delivery: evidence from an integrative literature review. Human Resources Health. 2013;11:51.

15. Bookman L, Engmann C, Srofenyoh E, Enweronu-Laryea C, Owen M, Randolph G, et al. Educational impact of a hospital-based neonatal resuscitation program in Ghana. Resuscitation. 2010;81(9):1180-2.

16. Kim YM, Ansari N, Kols A, Tappis H, Currie S, Zainullah P, et al. Assessing the capacity for newborn resuscitation and factors associated with providers' knowledge and skills: a cross-sectional study in Afghanistan. BMC Pediatr. 2013;13:140

17. Coffey P KL, Narayanan I, Lockwood JB, Singhal N, Wall S, Johnson J, Schoen E. Case Study: Newborn Resuscitation Devices. Working Paper Prepared for The United Nations Commission on Life-Saving Commodities for Women and Children 2012 19th July 2018]. Available from: http://www. everywomaneverychild.org/images/UN_Comission_Report_Resuscitation_ Devices_COMPLETE_reduced.pdf.

18. Reisman J, Arlington L, Jensen L, Louis H, Suarez-Rebling D, Nelson BD. Newborn Resuscitation Training in Resource-Limited Settings: A Systematic Literature Review. Pediatrics. 2016;138(2):e20154490.

19. World Health Organisation. Second biennial Progress report 2016-2017 (action plan for healthy newborn infants in the Western Pacific region: 2014-2020). Manila: World Health Organization Regional Office for the Western Pacific; 2018.

20. Musafili A, Essen B, Baribwira C, Rukundo A, Persson LA. Evaluating helping babies breathe: training for healthcare workers at hospitals in Rwanda. Acta Paediatr. 2013;102(1):e34-8.

21. Carlo WA, Wright LL, Chomba E, McClure EM, Carlo ME, Bann CM, et al. Educational impact of the neonatal resuscitation program in low-risk delivery centers in a developing country. J Pediatr. 2009;154(4):504-8.e5.

22. Van Heerden C, Maree C. Janse van Rensburg ES. Strategies to sustain a quality improvement initiative in neonatal resuscitation. Afr. 2016;8(2):e1-e10.

23. Kc A, Wrammert J, Nelin V, Clark RB, Ewald U, Peterson S, et al. Evaluation of helping babies breathe quality improvement cycle (HBB-QIC) on retention of neonatal resuscitation skills six months after training in Nepal. BMC Pediatr. 2017:17(1):103.

24. Silvestre MAA, Mannava P, Corsino MA, Capili DS, Calibo AP, Tan CF, et al. Improving immediate newborn care practices in Philippine hospitals: impact of a national quality of care initiative 2008-2015. Int J Qual Health Care. 2018;30(7):537-44.

25. Singhal N, Lockyer J, Fidler H, Keenan W, Little G, Bucher S, et al. Helping babies breathe: global neonatal resuscitation program development and formative educational evaluation. Resuscitation. 2012;83(1):90-6.
26. Namazzi G, Waiswa P, Nakakeeto M, Nakibuuka VK, Namutamba S, Najjemba $M$, et al. Strengthening health facilities for maternal and newborn care: experiences from rural eastern Uganda. Glob Health Action. 2015;8:24271.

27. Bell DS, Harless CE, Higa JK, Bjork EL, Bjork RA, Bazargan M, et al. Knowledge retention after an online tutorial: a randomized educational experiment among resident physicians. J Gen Intern Med. 2008;23(8):1164-71.

\section{Publisher's Note}

Springer Nature remains neutral with regard to jurisdictional claims in published maps and institutional affiliations.
Ready to submit your research? Choose BMC and benefit from:

- fast, convenient online submission

- thorough peer review by experienced researchers in your field

- rapid publication on acceptance

- support for research data, including large and complex data types

- gold Open Access which fosters wider collaboration and increased citations

- maximum visibility for your research: over $100 \mathrm{M}$ website views per year

At BMC, research is always in progress.

Learn more biomedcentral.com/submissions 